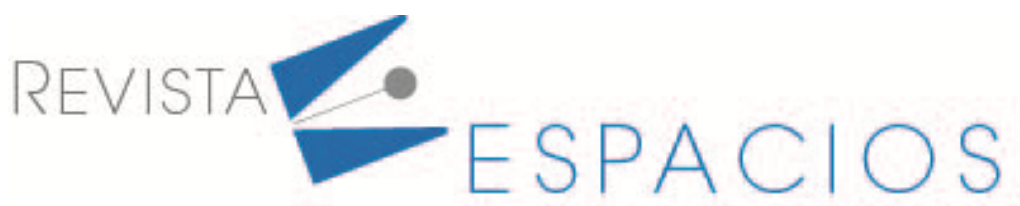

\title{
Competencias de liderazgo asociadas a la gestión del clima laboral: caso Universidad Politécnica Estatal del Carchi (Ecuador)
}

\section{Leadership competencies associated with the management of the work environment: case of the Universidad Politécnica Estatal del Carchi (Ecuador)}

PAGUAY, Félix W. ${ }^{1}$

\begin{abstract}
Resumen
El objetivo de esta investigación es determinar los factores de liderazgo de las autoridades de la Universidad Politécnica Estatal del Carchi (UPEC) asociados al clima organizacional, para lo cual se elaboró un modelo teórico conceptual con las competencias de liderazgo administrativas, interpersonales y conceptuales como factores de análisis. Se utilizó modelaje con ecuaciones estructurales y se encontró que los factores más importantes asociados con el clima organizacional son: la dirección de personas, la inteligencia emocional y la solución de problemas.

Palabras clave: clima organizacional, factores de liderazgo, liderazgo, líderes institucionales.
\end{abstract}

\begin{abstract}
The objective of this research is to determine the leadership factors of the authorities of the Universidad Politécnica Estatal del Carchi (UPEC) associated with the organizational climate, for which a theoretical conceptual model was elaborated with administrative, interpersonal and conceptual leadership competencies as factors of analysis. Modeling with structural equations was used and it was found that the most important factors associated with the organizational climate are: people management, emotional intelligence and problem solving.

Key words: organizational climate, leadership factors, leadership, institutional leaders.
\end{abstract}

\section{Introducción}

El clima organizacional no constituye un término nuevo dentro de la gestión, sin embargo, el interés de los académicos por esta temática se renueva, convirtiéndose en un pilar de investigación prioritario, por ejemplo, en el campo de la gestión estratégica. Si de pilares se trata los académicos dejan de lado su propia organización, las universidades como forjadoras de valores y principios sociales frecuentemente se ven afectadas por los cambios del entorno, por lo tanto, los departamentos de planificación estratégica deben alinear las operaciones y garantizar su permanencia con elevados estándares de calidad, lo cual sugiere que deben existir dos condiciones: 1 ) liderazgo competente y 2 ) un clima organizacional que refleje el compromiso de la universidad

\footnotetext{
${ }_{1}^{1}$ Profesor Investigador Titular. Escuela de Administración de Empresas. Universidad Politécnica Estatal del Carchi. Ecuador. felix.paguay@upec.edu.ec / felixpaguay@hotmail.es
} 
con la sociedad. En ese sentido, cabe preguntarse ¿qué tan importante resulta el clima organizacional para Instituciones de Educación Superior (IES)?, y ¿de qué depende un buen clima organizacional? Para responder estas interrogantes es preciso decir que los pioneros Lewin, Lippitt y White (1939) establecieron diferentes tipos de liderazgo a partir del comportamiento de los colaboradores en una organización, la interacción entre los integrantes del equipo constituye el principio básico del clima organizacional, "que puede entenderse como el ambiente que generan las emociones de un determinado grupo y en el cual influyen varios elementos" (Sokol, Gozdek, Figurska, y Blaskova, 2015, p.279).

Las instituciones de educación superior se constituyen en organizaciones con una alta responsabilidad social, que deben responder a las necesidades del entorno con la fortaleza que les da el clima organizacional. De acuerdo con autores como Thomas y Fredericks (1988); Tiwari (2014) y Cheng (2015) el buen clima organizacional en las instituciones de educación superior es un tema prioritario que influye en: la investigación, la enseñanza, la cooperación en investigación, la justicia organizacional y la internacionalización e innovación en el aprendizaje; además, afirman que las instituciones que desarrollan climas más positivos reportan una mayor claridad de objetivos y estándares de rendimiento.

La Universidad Politécnica Estatal del Carchi (UPEC), es una institución de educación superior que, desde su creación en el año 2006, asumió grandes retos y su gestión ha estado orientada a ofrecer una educación superior de calidad y excelencia. Como se mencionó con anterioridad, el clima organizacional es un factor determinante para cumplir los propósitos de cualquier tipo de organización, y en el caso de la UPEC no puede ser la excepción.

El clima organizacional se compone de algunas dimensiones, entre ellas se considera el liderazgo que según Natario, Araújo, y Couto (2014, p.107) tiene una influencia crítica sobre el clima organizacional, pues el líder debe ser capaz de hacer que el clima organizacional sea propicio para aumentar la productividad; también, menciona que el clima organizacional de una institución de educación superior debe contemplar la importancia de la influencia en la relación líder-colaborador.

Siendo un factor de gran impacto para el clima organizacional la influencia positiva del líder o líderes de una organización, la presente investigación procura determinar el impacto de dicha influencia desde las competencias analizadas en los líderes de la UPEC, partiendo de la hipótesis de que las competencias de liderazgo administrativas, interpersonales y conceptuales desarrolladas por los directivos, influyen de forma positiva en el clima organizacional de esta institución.

\subsection{Liderazgo}

"El estudio del liderazgo tiene una larga historia interdisciplinaria que se remonta a siglos e incluso milenios" (Perruci y McManus, 2012). A lo largo de la década de 1960, los principales teóricos de la organización consideraron el concepto de liderazgo como digno de una investigación intelectual seria. Académicos como Weber, Barnard, y Selznick creían que no se podía entender completamente lo que creen las personas en las organizaciones o cómo se comportan sin hacer referencia a la presencia (o ausencia) de los líderes de la organización (Podolny, Khurana, y Besharov, 2004).

El liderazgo como tal no se conceptualiza como una cosa única o un conjunto definitivo de prácticas, por el contrario, el liderazgo puede responder a múltiples demandas (Mackillop, 2018). Para contribuir de forma positiva en la organización el liderazgo debe ser auténtico (Crawford, Dawkins, Martin, y Lewis, 2019), esto por cuanto los miembros de una organización suelen adoptar un comportamiento de liderazgo demostrado por el líder (Grille, Schulte y Kaufeld, 2015). 
El liderazgo claramente tiene un impacto en los resultados de las organizaciones (Bischak y Woiceshyn, 2015), una de las ventajas de un buen liderazgo radica en mejorar el comportamiento y desempeño de los seguidores (Kock, Mayfield, Mayfield, Sexton, y Garza, 2018). Los líderes pueden influir positivamente en las actitudes y conductas laborales de los seguidores por diferentes medios (Burch y Guarana, 2014), sin embargo, son los comportamientos del líder los que deben influir de manera efectiva en sus colaboradores a fin de lograr los objetivos, (Kinicki, Jacobson, Galvin, y Prussia, 2011).

Entre las múltiples definiciones de liderazgo, está la de Juan Bravo, quien explica que el liderazgo puede entenderse como el hecho de: "lograr que las cosas sucedan también a través de los demás, en armonía con el bien común y con uno mismo" (2011, p. 13).

Chiavenato (2011), por su parte, considera que toda estrategia requiere del liderazgo, que puede ser entendido como "compromiso, implicación, espíritu misionero y visionario, enfoque en los objetivos, sentido de oportunidad y por extensión, énfasis en la participación y la dedicación de todos, en proporcionar impulso, orientación, refuerzo, realimentación, motivación y enfoque en el aprendizaje de las personas" (p. 244).

Brian Tracy, experto en éxito, advierte sobre la necesidad de liderazgo en nuestra sociedad, en nuestros hogares, en nuestras organizaciones, en nuestros negocios, en nuestras asociaciones públicas y privadas, en nuestro gobierno. “(...) necesitamos gente que tenga visión y valentía, gente con la capacidad de surcar nuevos mares y abrir nuevos caminos" (2015, p. 1).

\subsection{Factores de liderazgo}

Almuiñas y Galarza consideran que "el liderazgo constituye una variable relevante para el desarrollo de la dirección estratégica, pues los líderes trasmiten sus creencias y visión a los miembros de la organización" (2014, p. 47).

En este estudio se consideró como factores la agrupación de las competencias de liderazgo realizada por Northouse, quien divide a las competencias de liderazgo en tres categorías: administrativas, interpersonales y conceptuales (Palomo, 2013, p. 52).

Figura 1

Modelo de Competencias de liderazgo

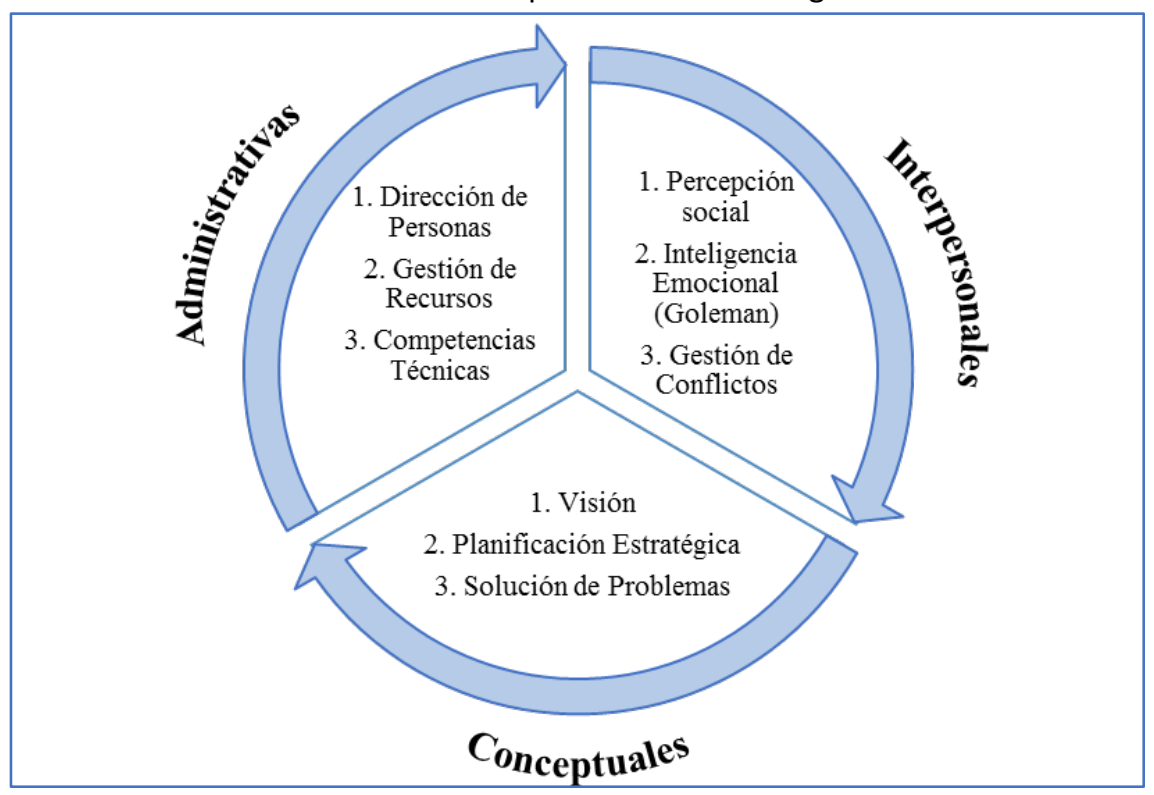

Fuente: Tomado de Palomo (2014). 


\subsubsection{Competencias administrativas o de gestión}

De acuerdo con Northouse (2009), estas competencias se refieren a las que posee el líder cuando tiene que conseguir objetivos o fines dentro de una organización, lo cual le exige la planificación, organización del trabajo, asignación adecuada de tareas y coordinación de actividades. "Estas competencias se subdividen en tres categorías: dirección de personas, gestión de recursos y competencias técnicas” (Palomo, 2013, p.52).

En la competencia de dirigir personas, un líder requiere alcanzar conexión con sus colaboradores. Comprende perfectamente las tareas de cada uno. Conoce incluso quiénes necesitan formación para mejorar su desempeño. Conoce perfectamente el ambiente de trabajo. "Participa activamente ayudando a fortalecer el trabajo en equipo, la motivación para mejorar los resultados, promover relaciones positivas y satisfactorias entre los miembros del su equipo, así como ofrece ayuda a quienes lo requieran" (Palomo, 2013, p.53).

Crea condiciones y escenarios para atraer, retener y comprometer a personas con talento. "Necesita comunicarse efectivamente con el consejo de administración, el equipo directivo, mandos medios de la organización, personas externas de organizaciones públicas o privadas, stakeholders"' (Palomo, 2013, p.53).

Por otro lado, la competencia de gestión de recursos requiere que el líder dedique tiempo a la gestión de todo tipo de recursos que permitan el perfecto funcionamiento de una organización, tanto tangibles (personas, recursos económicos, equipos, espacios, etc.) como intangibles (imagen de la empresa, el conocimiento tecnológico, el capital humano, la estructura organizativa, la marca, el logotipo o las relaciones con proveedores y clientes). "Entre las acciones están, solicitudes de equipos, búsqueda de trabajo, localización de fondos para proyectos" (Palomo, 2013, p.53).

Por su parte, las competencias técnicas sugieren que el líder debe tener conocimientos técnicos o comprender los aspectos más complejos sobre cómo hacer, o bien conocer quiénes pueden hacer algo de manera efectiva. Además, comprender la idiosincrasia del funcionamiento de la organización. "Es decir que el líder posee las competencias técnicas y las actividades que desempeñan sus colaboradores" (Palomo, 2013, p. 53).

\subsubsection{Competencias interpersonales}

Los estudios demuestran que estas competencias permiten a los líderes ser más efectivos con sus colaboradores. Se clasifican, según Palomo (2013), en percepción social, inteligencia emocional y gestión de conflictos. La percepción social se refiere a la sensibilidad sobre el impacto que tienen las ideas sobre los demás. Esto implica tener conocimiento profundo de lo que es importante para los demás, sus motivaciones, problemas y reacciones frente a los cambios, así como las necesidades, objetivos y demandas de las organizaciones. "Estos líderes transforman de manera efectiva a las organizaciones, equilibrando los impactos de los cambios, los cuales los gestionan de manera personalizada" (Palomo, 2013, p.54).

La inteligencia emocional puede entenderse como la comprensión de las emociones propias y las de los demás, es decir, percibir y expresar emociones, usar esas emociones para facilitar las cogniciones o pensamientos y gestionar las emociones de forma efectiva en las relaciones con los demás (Palomo, 2013, p. 55). Y, finalmente, la gestión de conflictos, entendidos como la lucha entre dos o más individuos sobre las diferentes percepciones acerca de un asunto o cuestión. De acuerdo con Palomo (2013) "la resolución de un conflicto reduce el estrés, incrementa la creatividad en la solución de problemas y mejora las relaciones entre el equipo y el líder" (p. 62).

\subsubsection{Competencias conceptuales}

Las competencias conceptuales se refieren a pensamientos o cogniciones inherentes al liderazgo. Según Palomo (2013), se dividen en: solución de problemas, planificación estratégica y visión ( p. 62). La solución de problemas se refiere a las habilidades cognitivas del líder para adoptar opciones correctas frente a un problema o de mejora 
de los objetivos deseados. "Incluye habilidades para identificar un problema, generación de alternativas, selección de alternativas idóneas y puesta en marcha de solución” (Palomo, 2013, p.62).

La planificación estratégica es a la capacidad de pensar, estudiar, desarrollar estrategias, planes, acciones, basados en recursos y talento humano disponible. Además, la necesidad de desarrollar habilidades de aprender, experimentar nuevas ideas incluso fracasos, "adaptarse, ser capaz de responder a los cambios del entorno, estar abierto y aceptar los cambios, es decir, tener profundo conocimiento de las personas y el entorno, es decir, la sabiduría de gestionar" (Palomo, 2013, p.62). Y, por último, la visión que consiste en la capacidad de proyectar una imagen del futuro para mejorar la situación actual y transmitir a los demás los nuevos ideales y valores que nos conducen a ese futuro.

\subsection{Clima Organizacional}

Según Cloete (2011), "la teoría del clima organizacional sugiere que el liderazgo en las organizaciones tiene un efecto significativo en la determinación del clima organizacional" (citado por Eustace y Martins, 2014, p.4).

A través del tiempo, el clima organizacional se ha convertido en un tema prioritario para todas las organizaciones. Bordas (2016) indica que sus inicios se remontan a investigaciones realizadas por Lewin, Lippit y White (1939), quienes identifican que el comportamiento del individuo depende de la atmósfera o clima social en el que está inmerso.

Según Castro y Martins (2010, p.1), las organizaciones en el siglo XXI enfrentan más desafíos que nunca. Estos desafíos no son exclusivos y afectan a todas las organizaciones, independientemente de su estructura o tamaño. El clima organizacional en particular es un desafío constante, ya que existen muchos cambios que afectan a las organizaciones hoy.

Un hito importante sobre el clima organizacional es la publicación del libro de McGregor "The Human Side of Enterprise" (1960), aquí el autor enfatiza sobre la importancia de los líderes en la creación del clima de trabajo. A partir de ello el concepto de clima organizacional ha ganado prominencia y su conceptualización hasta la fecha ha sido analizada por varios autores (Guediri y Griffin, 2015).

Para Chiavenato "El ambiente entre los miembros de la organización se llama clima organizacional y está estrechamente ligado al grado de motivación de las personas" (2009). Ilián (2011, p.41) considera que "el clima organizacional, en general, se relaciona con la atmósfera de trabajo que consiste en formas y métodos de funcionamiento organizacional asumido por los miembros de la organización".

El clima organizacional es uno de los conceptos principales en el comportamiento organizacional. Un clima organizacional adecuado conduce a la innovación e inspiración organizacional y tiene un papel positivo en el cumplimiento de los objetivos organizacionales. En consecuencia, los gerentes siempre deben monitorear el clima organizacional (Bahrami, Barati, Ghoroghchian, Montazer, y Ezzatabadi, 2016, p.97)

El estudio del clima organizacional en las Instituciones de Educación Superior resulta importante, de acuerdo con Thomas y Fredericks (1988, p.367), permite identificar en qué departamentos académicos o subunidades el clima organizacional es significativamente más positivo, pues consideran que estas áreas reportan una mayor claridad de objetivos y estándares de rendimiento. Por otra parte, Segredo (2011, p.164) “destaca la valoración del clima organizacional como una valiosa herramienta diagnóstica en la gestión del cambio, para una mayor eficiencia en las instituciones". 


\subsection{Dimensiones del clima organizacional}

Rodríguez, Álvarez, Sosa, Vos, Bonet y Van (2010) sugieren que el clima organizacional consta de las siguientes dimensiones: motivación compromiso y participación. La motivación tiene un papel central en el logro de altos rendimientos dentro de las organizaciones. "El clima organizacional puede estar estrechamente relacionado con la motivación de los empleados, ya que proporciona un entorno motivador en las empresas y depende de la capacidad de los gerentes para crear un clima organizacional de apoyo" (Rusu y Avasilcai, 2014, p.51).

Por otro lado, el uso del término compromiso organizacional (OC) se remonta a los años cincuenta del siglo XX como una preocupación básica de los gerentes (Aviad, 2019, p.13). Para Valaei y Rezaei (2016), no hay factores humanos ni construcciones que afecten los resultados organizacionales más que el compromiso organizacional. El concepto de compromiso organizativo indica la forma en que un miembro de la empresa está vinculado y tiene cierto grado de lealtad hacia la organización. Las definiciones de compromiso divergen y en su mayoría dependen del contexto organizacional ya que los académicos proporcionan diferentes definiciones y clasificaciones.

El compromiso organizacional se ha convertido en un concepto importante en la investigación organizacional y en la comprensión del comportamiento del personal en el trabajo porque refleja la medida en que el personal se identifica con la organización y se compromete con sus objetivos. El personal comprometido puede mejorar el crecimiento y la excelencia en la organización. Uno de los factores organizacionales que tiene un impacto significativo en el clima organizacional es el compromiso del personal (Bahrami, et al, 2016, p.97).

Por su parte, "la participación activa, decidida y voluntaria del personal es imprescindible para alcanzar altos niveles de satisfacción de los clientes" (Alcaide, 2015, p.183). La organización de una institución de Educación Superior debe construirse sobre la base de una conciencia participativa generalizada. Para lograr esta conciencia participativa se debe conocer su importancia, pues de acuerdo con Rodríguez, et al (2010) "la participación en la organización debe ser comprendida profundamente por todas y cada una de las personas involucradas, es el elemento esencial del proceso de integración" (p.187).

Si bien se han desarrollado varias investigaciones sobre el liderazgo y el clima organizacional, continúa el debate sobre la construcción del clima organizacional y el papel del liderazgo en la creación del clima deseado (Eustace y Martins, 2014).

El adecuado liderazgo en términos de gestión del clima organizacional, se compone de una serie de competencias y esas competencias se aprenden, por ello la importancia de conocerlas y fomentarlas entre los líderes empresariales. Siendo las Instituciones de Educación Superior, organizaciones complejas, el clima organizacional puede verse afectado por la falta de liderazgo, por ello, se debe destacar que es el líder "quien representa un factor fundamental para crear un clima organizacional que genere satisfacción y compromiso entre los empleados" (Peña, Parra, y Beltrán, 2014).

\section{Metodología}

Este trabajo de investigación tiene un enfoque cuantitativo y es de tipo descriptivo y correlacional. Se realizó un censo, donde el objeto de estudio está representado por 128 colaboradores (docentes) de los líderes de la UPEC en el año 2018. Las variables contempladas para la construcción del modelo teórico conceptual, surgieron de la revisión teórica, donde las variables independientes son las competencias de liderazgo: 1) competencias administrativas, 2) competencias conceptuales y 3) competencias interpersonales. Por su parte la variable dependiente corresponde al clima organizacional de la UPEC, medida por las siguientes categorías: a) motivación, b) compromiso, y c) participación. Por tanto, el modelo conceptual que surge en esta etapa, y que será sometido a comprobación, se presenta en la figura 2. 
Figura 2

Modelo teórico conceptual

sobre clima organizacional - UPEC

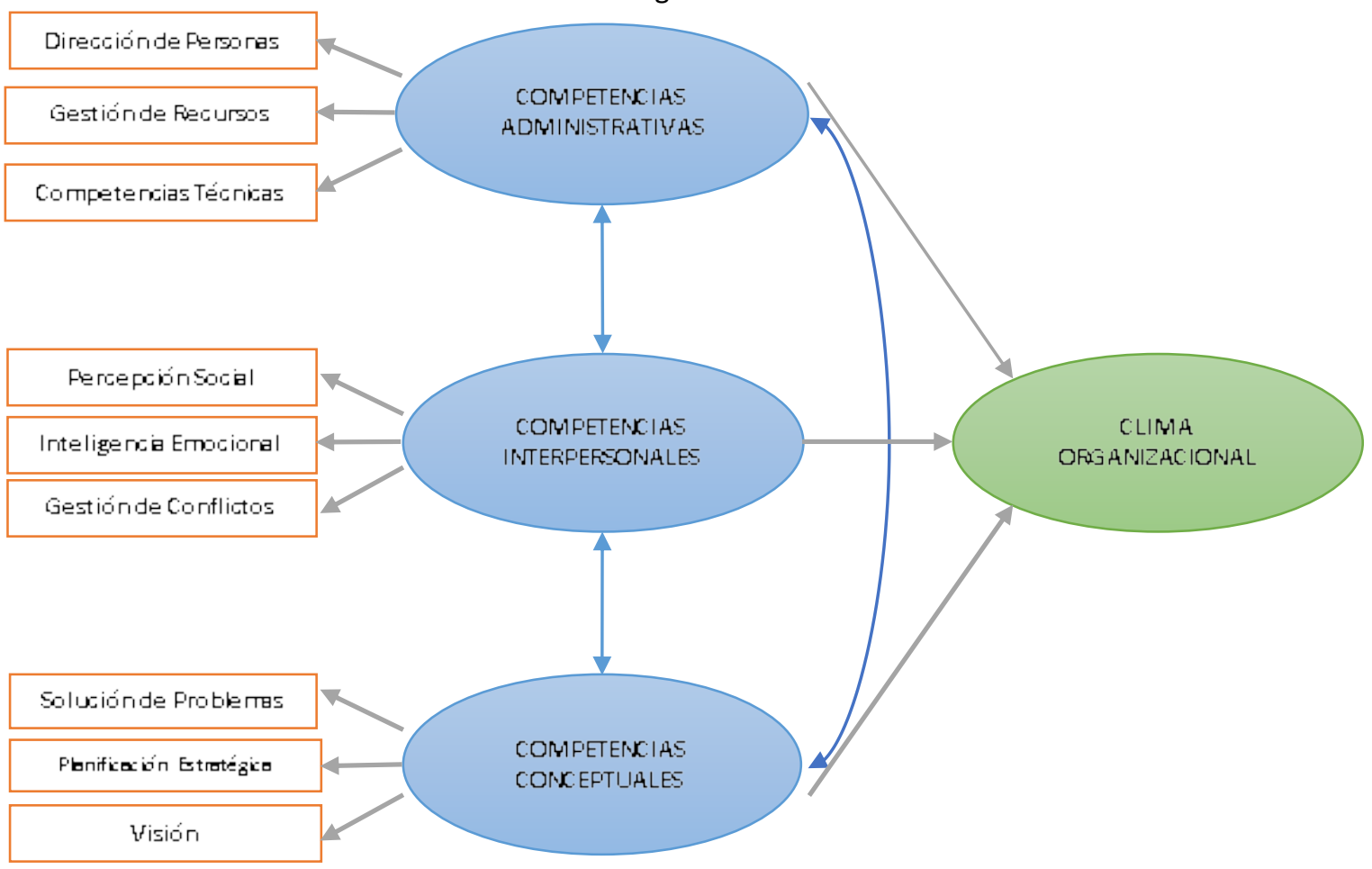

Fuente: Elaboración propia.

La hipótesis nula de esta investigación sostiene que las competencias de liderazgo están relacionadas directamente con el clima organizacional de la Universidad Politécnica Estatal del Carchi.

\subsection{Modelo de clima organizacional UPEC}

En este modelo, las competencias de liderazgo son variables latentes, razón por la que no pueden medirse de manera directa. La tabla 1 muestra cada una de las competencias de liderazgo, con sus respectivas variables medibles.

\section{Tabla 1}

Factores del Modelo de clima organizacional UPEC

\begin{tabular}{|c|c|}
\hline Factores & Categorías \\
\hline Competencias Administrativas & $\begin{array}{ll}\text { - } & \text { Dirección de personas } \\
\text { - } & \text { Gestión de Recursos } \\
\text { - } & \text { Competencias Técnicas }\end{array}$ \\
\hline Competencias Interpersonales & $\begin{array}{ll}\text { - } & \text { Percepción Social } \\
\text { - } & \text { Inteligencia Emocional } \\
\text { - } & \text { Gestión de Conflictos }\end{array}$ \\
\hline Competencias Conceptuales & $\begin{array}{ll}\text { - } & \text { Solución de Problemas } \\
\text { - } & \text { Planificación Estratégica } \\
\text { - } & \text { Visión }\end{array}$ \\
\hline
\end{tabular}

Fuente: Elaboración propia

Para poder comprobar el modelo teórico conceptual sobre el clima organizacional, se diseñó una encuesta como instrumento de recolección de información, la cual está compuesta por 33 preguntas que corresponden a la 
variable independiente y 33 a la variable dependiente. Con un total de 66 ítems, las opciones de respuesta corresponden a una escala de Likert codificadas de la siguiente manera: totalmente en desacuerdo $=1$, bastante en desacuerdo $=2$, ni acuerdo, ni en desacuerdo $=3$, bastante de acuerdo $=4$, y totalmente de acuerdo $=5$.

La validación del instrumento de investigación se realizó a través de la aplicación de 20 encuestas piloto, donde el resultado del coeficiente de alfa de Cronbach, fue de .982. Para la construcción de los factores se utilizó la revisión de la literatura y el análisis factorial, el criterio para la extracción de factores fue de acuerdo con el propuesto por Hair, Anderson, Black, y Babin (2016), es decir que se tomaron aquellos factores que arrojaron un auto valor mayor a 1 (ver tabla 2). El análisis arrojó una medida de adecuación muestral de Kaiser- Meyer-Olkin= 0.938 .

Tabla 2

Resultados del Análisis Factorial

\begin{tabular}{|l|c|c|}
\hline \multicolumn{1}{|c|}{ Factor } & Autovalor & Varianza Explicada \\
\hline Competencias Administrativas & 47.758 & 63.270 \\
\hline Competencias Interpersonales & 1.999 & 66.299 \\
\hline Competencias Conceptuales & 1.425 & 68.459 \\
\hline Motivación & 1.286 & 70.408 \\
\hline Compromiso & 1.189 & 72.210 \\
\hline Participación & 1.036 & 73.780 \\
\hline
\end{tabular}

Fuente: Elaboración propia

Luego el instrumento validado fue aplicado a un total de 128 docentes que integran el objeto de estudio. Finalmente, los datos obtenidos fueron analizados utilizando la técnica estadística de análisis multivariante «modelaje con ecuaciones estructurales» en el software estadístico AMOS.

\section{Resultados}

De acuerdo con Tabachnick y Fidell (2013, p.722), se debe considerar el índice CFI de bondad de ajuste del modelo que varía entre 0-1, donde 1 significa un ajuste perfecto, 0,95 ajuste óptimo y 0,9 un ajuste satisfactorio; para este caso este índice tiene un resultado de 0,987, es decir la bondad de ajuste del modelo es óptima. En la figura 3 se ofrece un breve resumen de los resultados que arroja el modelo empírico respecto a las competencias de liderazgo y su relación con el clima organizacional.

Cabe aclarar que debido al tamaño de la población (128), se tomaron algunas medidas para evitar problemas de colinealidalidad y otro tipo de inconsistencias estadísticas. Primero, el índice No normalizado de ajuste (NNFI), o índice Tucker Lewis para considerar un ajuste optivo debe ser mayor a 0.90 (Escobedo, Hernández, Estebané y Martínez, 2016), para el caso de esta investigación el NNFI fue de 0.92, este índice se emplea cuando la relación del modelo es débil con el tamaño de la muestra. 
Figura 3

Modelo empírico sobre clima organizacional - UPEC

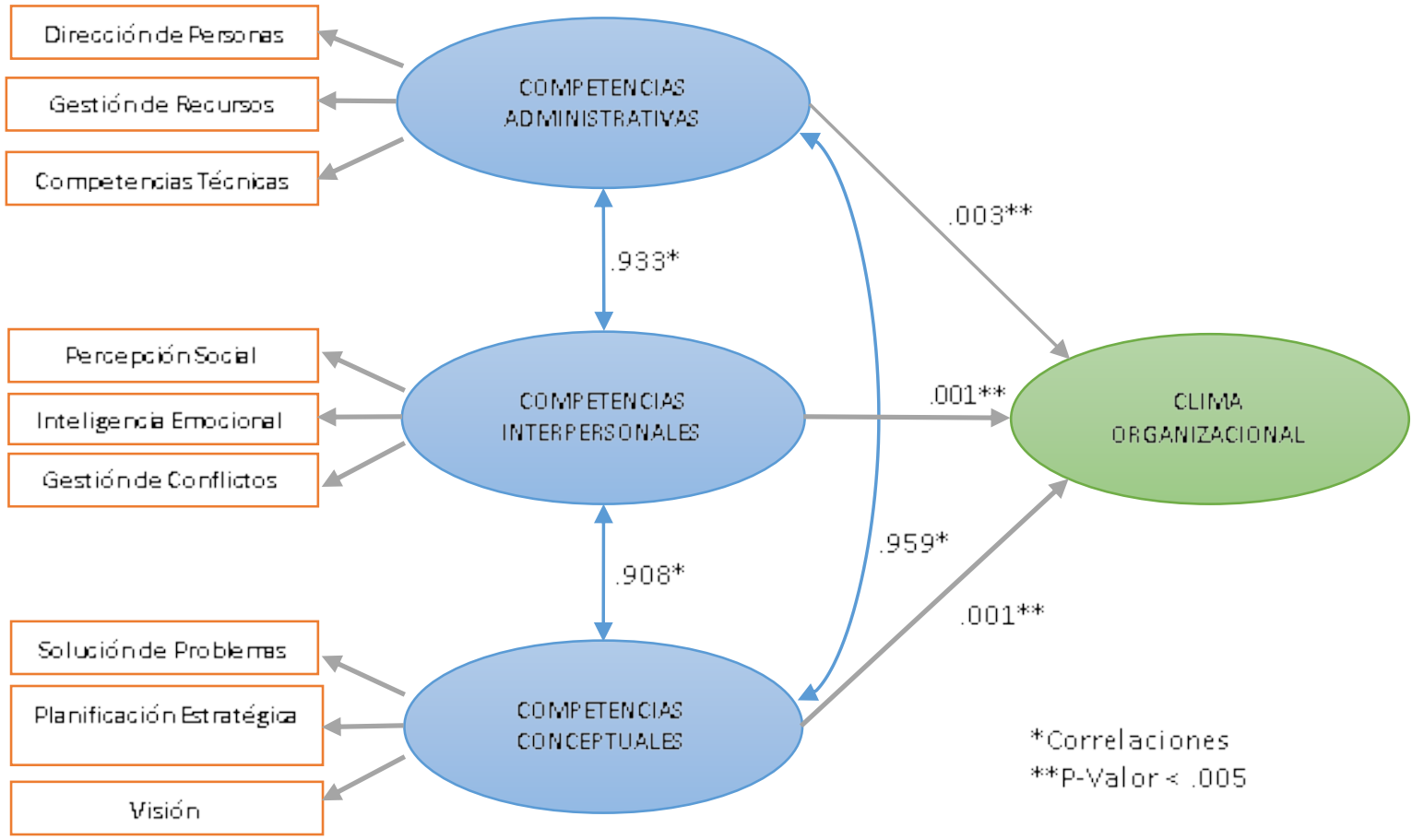

Fuente: Elaboración propia.

Las asociaciones entre las variables independientes con respecto a la variable dependiente son directas, puesto que, todas las competencias de liderazgo como factores tienen un p-valor menor a .005 (tabla 3).

Tabla 3

Asociación entre competencias de liderazgo y clima organizacional - UPEC

\begin{tabular}{|l|c|c|}
\hline \multicolumn{1}{|c|}{ Asociación } & Competencias & Valor P \\
\hline Clima Organizacional & Administrativas & .003 \\
\hline Clima Organizacional & Interpersonales & .001 \\
\hline Clima Organizacional & Conceptuales & .001 \\
\hline
\end{tabular}

Fuente: Elaboración propia.

Por otra parte, los factores representados por las competencias de liderazgo, presentan una correlación significativa entre sí (ver tabla 4), lo anterior valida las proposiciones de Northouse (2009) y Palomo (2013) respecto al conjunto de competencias que debe dominar el líder.

\section{Tabla 4}

Correlación entre las competencias del modelo de clima organizacional - UPEC Asociación entre competencias

\begin{tabular}{|l|r|}
\hline Competencias Administrativas & Competencias Interpersonales \\
\hline Competencias Administrativas & Competencias Conceptuales \\
\hline Competencias Interpersonales & Competencias Conceptuales \\
\hline & Fuente: Elaboración propia.
\end{tabular}

\section{Correlaciones}

En la tabla 5 se muestran las variables dentro del factor competencias administrativas que tuvieron pesos estandarizados más elevados, estos fueron aquellos relacionados con: adaptación, ayuda, admisión, información y accesibilidad. 
Tabla 5

Pesos estandarizados de las variables respecto a las Competencias Administrativas

\begin{tabular}{|l|l|r|}
\hline \multicolumn{1}{|c|}{ Variables } & \multicolumn{1}{c|}{ Factor } & Peso Estandarizado \\
\hline ADAPTA & Competencias Administrativas & .864 \\
\hline AYUDA & Competencias Administrativas & .851 \\
\hline ADMITE & Competencias Administrativas & .845 \\
\hline INFORMA & Competencias Administrativas & .828 \\
\hline ACCESIBLE & Competencias Administrativas & .823 \\
\hline
\end{tabular}

Fuente: Elaboración propia.

Las variables con pesos estandarizados elevados con respecto a las competencias interpersonales de los líderes de la UPEC se presentan a continuación:

Tabla 6

Pesos estandarizados de las variables respecto a las Competencias Interpersonales

\begin{tabular}{|l|l|c|}
\hline \multicolumn{1}{|c|}{ Variables } & \multicolumn{1}{c|}{ Factor } & Peso Estandarizado \\
\hline ENTUSIASMADO & Competencias Interpersonales & .913 \\
\hline DERRIBA & Competencias Interpersonales & .890 \\
\hline JUSTAS & Competencias Interpersonales & .888 \\
\hline GENERA & Competencias Interpersonales & .883 \\
\hline COMPRENDE & Competencias Interpersonales & .874 \\
\hline
\end{tabular}

Fuente: Elaboración propia.

Por último, en la tabla 7 se presentan las variables de mayor peso referentes a las competencias conceptuales de los líderes de la institución.

Tabla 7

Pesos estandarizados de las variables respecto a las Competencias Conceptuales

\begin{tabular}{|l|l|c|}
\hline \multicolumn{1}{|c|}{ Variables } & \multicolumn{1}{c|}{ Factor } & Peso Estandarizado \\
\hline OCUPA & Competencias Conceptuales & .888 \\
\hline PLANIFICA & Competencias Conceptuales & .836 \\
\hline COMPRUEBA & Competencias Conceptuales & .830 \\
\hline ILUSIONADO & Competencias Conceptuales & .830 \\
\hline FAVORECE & Competencias Conceptuales & .823 \\
\hline
\end{tabular}

Fuente: Elaboración propia.

\subsection{Discusión}

Con los resultados obtenidos se logró confirmar la hipótesis planteada, pues las competencias de liderazgo desarrolladas por los líderes según la percepción del cuerpo docente están relacionadas directamente con el clima organizacional de la institución y se logran bosquejar en el modelo propuesto en el presente trabajo. Por otra parte, las variables con elevados pesos estandarizados corresponden a las siguientes categorías:

Con respecto a las competencias administrativas, la percepción del cuerpo docente con mayor peso recae en la categoría dirección de personas, de forma específica en la adaptación, es decir, los líderes de la UPEC se adaptan sin problemas a cualquier situación y/o persona. En concordancia con Serra (2016), el líder que se adapta a su equipo y las diferentes situaciones resulta un líder visionario, instructor, consultor y facilitador; por otra parte, Pozo (2013, p.111) manifiesta que los líderes que adaptan su estilo de liderazgo para satisfacer las necesidades de una situación dada son eficaces. Estas consideraciones permiten deducir que las competencias de liderazgo 
administrativas desarrolladas por los líderes de la UPEC resultan positivas en la creación de un buen clima organizacional.

En las competencias interpersonales, la categoría de mayor peso es la inteligencia emocional, ya que, los docentes perciben que los líderes logran que el personal se sienta entusiasmado con su trabajo. La labor de los líderes va más allá de lograr alcanzar los objetivos; un líder debe ser consciente que el verdadero desafío está en mantener la armonía del equipo, hacer que cada colaborador se sienta entusiasmado por realizar su aporte en beneficio de todos (Maratea, 2013, p.51). Horowitz (2017) considera que al responder a la pregunta cestán tus empleados entusiasmados por trabajar? de forma positiva, resulta evidente la existencia de la motivación. Los líderes de la UPEC logran que sus colaboradores estén motivados y como resultado obtienen un clima organizacional adecuado, ya que la motivación está ligada estrechamente con el clima organizacional (Rusu y Avasilcai, 2014, p.51)

En el caso del factor competencias conceptuales, la categoría solución de problemas tiene mayor peso con la afirmación de que los líderes de la UPEC se preocupan y ocupan por gestionar los casos de bajo rendimiento. Para Maxwell (2018, p.126), la solución de problemas es la forma más rápida de alcanzar el liderazgo, pues ofrece al líder oportunidades únicas para demostrar sus capacidades. Además, Bhat (2016, p.445) afirma la existencia de una relación positiva y significativa entre el clima organizacional y el desempeño laboral de los profesionales de la enseñanza. En otras palabras, los líderes de la UPEC logran mejorar el clima organizacional cuando usan sus competencias conceptuales para dar solución a problemas relacionados con el bajo rendimiento de sus colaboradores.

Las categorías antes mencionadas agrupan las variables de estudio dadas por las competencias de liderazgo que desarrollan los líderes de la UPEC, mismas que influyen de forma significativa en el clima laboral de la institución y por tal razón deben ser consideradas como factores de protección. Sin embargo, a pesar de la existencia de las diferentes variables observables que resultan positivas para la creación de un buen clima laboral, existen algunas que por su bajo peso estandarizado con respecto a las demás se consideran como categorías de riesgo para las competencias de liderazgo, mismas que se muestran a continuación:

Tabla 8

Pesos estandarizados de las variables respecto a las Competencias Conceptuales

\begin{tabular}{|l|l|l|c|}
\hline \multicolumn{1}{|c|}{ Variables Latentes } & & Variables Observables & Peso Estandarizado \\
\hline Competencias Administrativas & TIEMPO & $\begin{array}{l}\text { Siento que me dedica el tiempo que } \\
\text { necesito }\end{array}$ & 0,723 \\
\hline Competencias Interpersonales & MUESTRA & $\begin{array}{l}\text { Muestra gran sensibilidad hacia } \\
\text { temas medioambientales. }\end{array}$ & 0,663 \\
\hline Competencias Conceptuales & ACTUACIÓN & $\begin{array}{l}\text { Su actuación profesional se basa en } \\
\text { principios éticos y morales. }\end{array}$ & 0,575 \\
\hline
\end{tabular}

Fuente: Elaboración propia.

\section{Conclusiones}

Las competencias de liderazgo administrativas, interpersonales y conceptuales desarrolladas por los líderes de la institución como el Rector, Vicerrector, Decanos, Directores y Jefes de Área, están relacionadas de forma directa con el clima organizacional, razón por la que se deben mantener y mejorar las competencias antes descritas con la finalidad de conservar un adecuado clima organizacional.

Se puede concluir que los líderes de la UPEC pueden fortalecer el clima organizacional si se enfocan en mantener las variables de protección de las diferentes factores de liderazgo como: la dirección de personas a través de la 
adaptación a situaciones y personas, la inteligencia emocional que permite mantener a los colaboradores entusiasmados por hacer su trabajo y la solución de problemas que se ve reflejada con mayor énfasis al preocuparse y ocuparse por gestionar los casos de bajo rendimiento.

Finalmente se puede concluir que a pesar de la existencia de algunos aspectos que favorecen cada competencia de liderazgo desarrollada en la institución, también existen ciertos aspectos con menor peso en comparación a otros, mismos que deben ser considerados por los líderes a fin de mejorarlos y contribuir en el perfeccionamiento del clima organizacional.

\section{Referencias bibliográficas}

Alcaide, J. (2015). Fidelización de Clientes. Madrid: ESIC Editorial.

Almuiñas, J., \& Galarza, J. (2014). Dirección estratégica y calidad en la Educación Superior. Durango: Editorial de la Universidad Juárez del Estado de Durango.

Aviad, H. (2019). Organizational Commitment: The Case of Unrewarded Behavior. Singapore: World Scientific. doi:10.1142/10768

Bahrami, M., Barati, O., Ghoroghchian, M., Montazer, R., \& Ezzatabadi, M. (2016). Role of Organizational Climate in OrganizationalCommitment: The Case of Teaching Hospitals. Osong Public Health and Research Perspectives, 96-100. doi:10.1016/j.phrp.2015.11.009

Bhat, S. (2016). Influence of organizational Climate on Job Performance of teaching professionals: An empirical study. International Journal of Education and Management Studies, 445-448.

Bischak, D., \& Woiceshyn, J. (2015). Leadership Virtues Exposed: Ethical Leadership Lessons From Leading in Rock Climbing. Journal of Leadership \& Organizational Studies, 23(3), 248-259.

doi:10.1177/1548051815617629

Bordas, M. (2016). Gestión Estratégica del Clima Laboral. Madrid: Editorial UNED.

Bravo, J. (2011). Liderazgo. Santiago de Chile: Evolución S. A. .

Burch, T., \& Guarana, C. (2014). The Comparative Influences of Transformational Leadership and Leader Member Exchange on Follower Engagement. Journal of Leadership Studies, 8(3), 6-25. doi:10.1002/jls.21334

Castro, M., \& Martins, N. (2010). The Relationship Between Organisational Climate and Employee Satisfaction in a South African Information and Technology Organisation. SA Journal of Industrial Psychology, 36(1), 19. doi:10.4102/sajip.v36i1.800

Cheng, Y. (2015). The Effects of Higher Education's Institutional Organizational Climate on Performance Satisfaction: Perceptions of University Faculty in Taiwan. International Business Research, 8(8), 103-117. doi:10.5539/ibr.v8n8p103

Chiavenato, I. (2009). Comportamiento Organizacional: La dinámica del éxito en las organizaciones. México: McGraw Hill.

Chiavenato, I. (2011). Planeación Estratégica. México: Mc Graw Hill. 
Crawford, J., Dawkins, S., Martin, A., \& Lewis, G. (2019). Putting the leader back into autenthic leadership: Reconceptualising and rethinking leaders. Australian Journal of Management, 1-21. doi:doi:10.1177/0312896219836460

Escobedo,María; Hernández, Jesús; Estebané, Virginia \& Martínez, Guillermina. (2016). Modelos de ecuaciones estructurales: Características, fases, construcción, aplicación y resultados. Cienc Trab. [Internet]. 18( 55 ): 16-22. Disponible en: https://scielo.conicyt.cl/scielo.php?script=sci_arttext\&pid=S071824492016000100004\&Ing=es. http://dx.doi.org/10.4067/S0718-24492016000100004.

Eustace, A., \& Martins, N. (2014). The role of leadership in shaping organisational climate: An example from the fast moving consumer goods industry. SA Journal of Industrial Psychology, 40(1), 1-13. doi:https://doi.org/10.4102/sajip.v40i1.1112

Grille, A., Schulte, E., \& Kaufeld, S. (2015). Promoting Shared Leadership: A Multilevel Analysis Investigating the Role of Prototypical Tea (Northouse, 2009)m Leader Behavior, Psychological Empowerment, and Fair Rewards. Journal of Leadership \& Organizational Studies, 1-16. doi:doi:10.1177/1548051815570039

Guediri, S., \& Griffin, M. (2015). Organizational Climate. En S. Clarke, T. Probst, F. Guldenmund, \& J. Passmore, The Wiley Blackwell Handbook of the Psychology of Occupational Safety and Workplace Health (págs. 272298). Estados Unidos de America: John Wiley \& Sons.

Hair, J., Anderson, R., Black, B., \& Babin, B. (2016). Multivariate Data Analysis. Unites States of America: Pearson Education.

Horowitz, B. (2017). Emprender y Liderar un Startup: El duro camino hasta el éxito. Barcelona: Libros de Cabecera.

Ilián, V. (2011). Competencias esenciales, clima organizacional e innovación como factores de competitividad empresarial: Propuesta y aplicación de un modelo para la detección y desarrollo de competencias en la Pequeña y Mediana Empresa del sector calzado en México. Universidad Autónoma de Madrid.

Kinicki, A., Jacobson, K., Galvin, B., \& Prussia, G. (2011). A Multilevel Systems Model of Leadership. Journal of Leadership \&Organizational Studies, 18(2), 133-149. doi:10.1177/1548051811399445

Kock, N., Mayfield, M., Mayfield, J., Sexton, S., \& Garza, L. (2018). Empathetic Leadership: How Leader Emotional Support and Understanding Influences Follower Performance. Journal of Leadership \& Organizational Studies, 1-20. doi:doi:10.1177/1548051818806290

Mackillop, E. (2018). Leadership in organisational change: A post-structuralist research agenda. Organization, 25(2), 205-222. doi:doi.org/10.1177/1350508417733137

Maratea, R. (2013). Liderar con Swing. Buenos Aires: Ediciones Granica.

Maxwell, J. (2018). Desarrolle el líder que está en usted 2.0. Estados Unidos de América: Grupo Nelson.

McGregor, D. (1960). The Human Side of Enterprise. New York: McGraw -Hill.

Northouse, P. (2009). Introduction to leadership. California: SAGE.

Natario, M., Araújo, C., \& Couto, J. (2014). Higher Education Institution Organizational Climate Survey. International Journal of Advances in Management and Economics, 3, 107-121.

Palomo, M. (2013). Liderazgo y motivación de equipos de trabajo. Madrid: ESIC Editorial. 
Peña, B., Parra, M., \& Beltrán, M. (2014). Liderazgo resonante. Un análisis a través de grupos de discusión. Historia y Comunicación Social , 143-151.

Perruci, G., \& McManus, R. (2012). The State of Leadership Studies. Journal of Leadership, 6(3), 49-54. doi:10.1002/jls. 21256

Podolny, J., Khurana, R., \& Besharov, M. (2004). Revisiting the Meaning of Leadership. Research in Organizational Behavior, 1-36. doi:10.1016/S0191-3085(04)26001-4

Pozo, J. D. (2013). Técnicas de Comunicación Personal y Grupal. Alicante: ECU.

Rodríguez, A., Álvarez, A., Sosa, I., Vos, P. D., Bonet, M., \& Van, P. (2010). Inventario del clima organizacional como una herramienta necesaria para evaluar la calidad del trabajo. Revista Cubana de Higiene y Epidemiología, 48(2), 177-196.

Rusu, G., \& Avasilcai, S. (2014). Linking Human Resources Motivation to Organizational Climate. Procedia Social and Behavioral Sciences, 51-58. doi:10.1016/j.sbspro.2014.02.459

Segredo, A. (2011). La gestión universitaria y el clima organizacional. Revista Cubana de Educacion Medica Superior, 25(2), 164-177.

Serra, J. (2016). Todo lo que debe saber sobre gestion de la farmacia. Liderar Personas. Barcelona: PROFIT Editorial.

Sokol, A., Gozdek, A., Figurska, I., \& Blaskova, M. (2015). Organizational climate of higher education institutions and its implications for the development of creativity. Procedia - Social and Behavioral Sciences, 279-288. doi:10.1016/j.sbspro.2015.04.767

Tabachnick, B., \& Fidell, L. (2013). Using Multivariate Statistics. United States of America: Pearson.

Thomas, E., \& Fredericks, J. (1988). Examining Organizational Climate in Institutions of Higher Education. Research in Higher Education, 28, 367-389. doi:10.1007/BF01006405

Tiwari, U. (2014). Organizational Climate In Higher Education. Abhinav International Monthly Refereed Journal of Research in Management \& Technology, 3(10), 1-6.

Tracy, B. (2015). Liderazgo. Nashville: Grupo Nelson.

Valaei, N., \& Rezaei, S. (2016). Job satisfaction and organizational commitment: An empirical investigation among ICT-SMEs. Management Research Review, 39(12), 1663-1694. doi:10.1108/mrr-09-2015-0216

Esta obra está bajo una Licencia Creative Commons Atribución-NoComercial 4.0 Internacional 